\title{
La interdisciplinariedad de las ingenierías, gestión del fortalecimiento de las capacidades en comunidades rurales
}

Diana Mabel Muñoz Enamorado ${ }^{1}$
Angela Gabriela Morales Santos $^{2}$
Fernando José Zorto Aguilera $^{3}$

\section{RESUMEN}

Las carreras de ingenierías juegan un rol muy importante en el desarrollo de las comunidades que buscan como resolver sus carencias y falta de recursos. La interdisciplinariedad es una ventaja real en toda la Facultad de Ingeniería de la Universidad Nacional Autónoma de Honduras. Esto tiene como beneficio el poder intervenir desde diversas áreas del conocimiento las problemáticas que existen en las zonas afectadas de una comunidad brindando un apoyo a la gestión de fortalecimiento de las capacidades de los pueblos indígenas en el país. El estudio plantea la manera de crear un modelo de intervención utilizando múltiples enfoques de las diferentes carreras de ingeniería de la UNAH para desarrollar una visión clara en su gestión en el apoyo de las capacidades a Las zonas rurales del país. La investigación hace constar que la integración de las diferentes carreras puede brindar las soluciones necesarias para el fortalecimiento de las capacidades de estas zonas. La ingeniería como área del conocimiento en la UNAH puede definir acciones interdisciplinarias reales para clarificar la gestión de apoyo con las comunidades, estructurando su intervención con base a las áreas disciplinarias para desarrollar actividades y soluciones a la problemática que tienen estas comunidades rurales.

Palabras clave: Interdisciplinariedad, Ingeniería, Vinculación, Investigación, capacidades

\footnotetext{
${ }^{1}$ Estudiante de la Carrera de Ingeniería Civil, Facultad de Ingeniería, UNAH: diana.munoz@unah.hn 2Estudiante de la Carrera de Ingeniería Civil, Facultad de Ingeniería, UNAH: angela.morales@unah.hn 3 Profesor de la Carrera de Ingeniería Mecánica, Facultad de Ingeniería, UNAH: fernando.zorto@unah.edu.hn
} 


\section{ABSTRACT}

Engineering careers play a very important role in the development of communities that seek to solve their shortcomings and lack of resources. Interdisciplinarity is a real advantage in all the engineering faculty, which has the benefit of being able to intervene from several areas of knowledge the problems that exist in the affected areas of an affected community, providing support to the management of capacity building of rural zone. The study sets out how to create a model of intervention using multiple approaches from the different engineering careers of the UNAH to develop a clear vision in its management in the support of capacities to the rural zone. The research shows that the integration of the different careers can provide the necessary solutions for the strengthening of the capacities of these rural zones. Engineering as a knowledge area at UNAH can define real interdisciplinary actions to clarify the management of support with the community, structuring its intervention based on the disciplinary areas to develop activities and solutions to the problems that these rural zones have.

Keywords: Interdisciplinarity, Engineering, Linkage, Research, capacities 


\section{INTRODUCCIÓN}

La Universidad Nacional Autónoma de Honduras (UNAH), a través de las carreras de la Facultad de Ingeniería, integra equipos interdisciplinarios para apoyar la gestión de fortalecimiento de las capacidades de las comunidades rurales de Yamaranguila, departamento de Intibucá. El artículo actual describe el plan de intervención que tienen los equipos interdisciplinarios en la comunidad de Carrizal Semane, a través de una metodología planteada para integrar a todas las carreras y sean ellas quienes ejecuten la acción de acompañamiento en la comunidad. Se llegó a la conclusión que la mejor manera de llegar dar apoyo a la gestión de fortalecimiento de la zona es a través de un modelo de comunidad sustentable.

\section{OBJETIVOS}

El objetivo del presente artículo sobre la interdisciplinariedad en las ingenierías es crear un modelo de intervención utilizando múltiples enfoques de las diferentes carreras de ingeniería de la UNAH para desarrollar una visión clara en su gestión en el apoyo de las capacidades a las comunidades rurales. Entre los objetivos específicos de la investigación se encuentran:

1. Integrar a las carreras de ingeniería de la UNAH para que desarrollen proyectos de investigación e innovación mediante el papel de la interdisciplinariedad para lograr una gestión de apoyo integral en las comunidades rurales.

2. Definir las acciones de interdisciplinariedad de las carreras de ingeniería de la UNAH para clarificar su gestión de apoyo en las comunidades rurales del país. A través de los conocimientos transmitidos en clases, desarrollarán procesos de investigación científica para encontrar soluciones claves dentro de los pueblos.

3. Estructurar las acciones de interdisciplinariedad de las carreras, de tal manera que sean éstas efectivas en función de las distintas áreas de conocimientos, las cuales serán útiles para el fortalecimiento de las capacidades de las comunidades rurales.

4. Desarrollar las actividades de los diferentes equipos interdisciplinarios organizados por los integrantes de las carreras de ingeniería de la UNAH según sea el proyecto de intervención seleccionado por ellos. 


\section{MARCO TEÓRICO}

La Universidad Nacional Autónoma de Honduras (UNAH), en su proceso de cambio planteado en la reforma educativa, trata de enfocarse en la manera de como propiciar el pensamiento crítico y profundo en la educación del hondureño, para que sea un actor estelar en el crecimiento científico y no meramente profesional, brindando soluciones creativas en las diversas instancias. En este caso, la universidad cuenta con facultades, departamentos y carreras que ayudan a desenvolverse de manera adecuada en los diferentes ámbitos de la academia (UNAH, 2014).

El nuevo modelo educativo de la universidad, deslumbra la metodología de cómo deben estar orientado los esfuerzos de las unidades académicas fundamentales, estos debe ser: a) Innovadoras; b) creativas; y c) con tendencia al cambio. Estas unidades deben buscar hacer énfasis en aprender a aprender, aprender a enseñar, aprender a hacer, aprender a ser, aprender a comunicarnos y convivir; donde se requiere que, el mismo, sea esencialmente investigativo (UNAH, 2009).

Según la UNAH (2009), en su modelo educativo hace mención que la realidad no puede verse desde una sola perspectiva, dado que dicha realidad tiende a ser más compleja de lo que es en realidad. Por esta razón, la universidad ha reflexionado que para abarcar y poder conocer las diferentes aristas de la realidad, la interdisciplinariedad es la vía adecuada para desarrollar su modelo. Pero Van Dusseldorp \& Wigboldus (2008) manifiestan que existe una fuerte dificultad en este proceso interdisciplinario: el ámbito de la investigación. Ellos describen que la comunidad científica no muestra un gran interés en las políticas de acción orientadas a la investigación interdisciplinaria. Son más relevantes para la comunidad científica los procesos disciplinarios de investigación.

Sin embargo Morillos, Bordons et al. (2003) discrepan en esta idea, mencionando que en las áreas de las ciencias duras, tales como las Ciencias Naturales, Física, Ingeniería, Matemática, Medicina y Química, se encuentran muy interesadas en el proceso interdisciplinario. El más aplaudido caso de las ingenierías, reafirmando la postura de Van de Besselaar \& Heimerik (2001) de que las carreras científico-tecnológicas son las que mayor aporte hacen a las políticas de interdisciplinariedad de una organización. Marques (2008) describe la interdisciplinariedad en diferentes carreras de ingeniería a nivel de pregrado y posgrado, destacando su importancia como iniciativa en una educación de ingeniería más dinámica. 
Para poder entender aún más este proceso, Dusseldorp \& Wigboldus (2008) brindan una tipología de la interdisciplinariedad, las cuales se encuentran en la Tabla 1.

Según el tipo de participantes, los proyectos interdisciplinarios pueden ser integrados por:

1. Disciplinas con el mismo paradigma o método. Es mucho más fácil integrar los miembros que contengan en sus áreas de conocimiento paradigmas o métodos similares en sus propias disciplinas. Sin embargo, cuando se integran con disciplinas con diferente paradigma, es mucho más difícil integrar las diferentes aportaciones al proyecto.

2. Un número significativo de disciplinas, lo cual conlleva a reducir o aumentar los problemas de comunicación entre las partes para brindar los entregables deseados.

3. Disciplinas de diferentes institutos u organizaciones. La dificultad de este tipo de proyecto es la organización y comunicación entre las personas involucradas que desarrollarán el proyecto. Por esta razón, se considera más fácil la interacción de miembros de la misma organización.

4. Miembros de diferente nacionalidad y cultura en estos casos, también surge el problema de comunicación.

Así mismo, estos mismos autores sugieren que para poder comenzar un equipo interdisciplinario, es necesario constituirlo con un número reducido de colaboradores con disciplinas que utilicen los mismos paradigmas, ligados a una organización y relacionados a los mismos aspectos culturales para su colaboración en el proyecto.

La gestión académica que se debe implementar como visión para realizar un proyecto de intervención para el fortalecimiento de los pueblos indígenas deberá estar ligada al desarrollo de la sustentabilidad de las zonas o pueblos indígenas. La sustentabilidad como ciencia ha de ser profundamente interdisciplinar, puesto que se asumen desafíos muy complejos para ser tratados desde la perspectiva aislada de una sola carrera. En dicha realidad interviene problemas diversos pero vinculados al accionar de múltiples disciplinas interconectadas (Gil-Perez \& Vilches, 2014). 


\section{Tabla 1. Tipos de Interdisciplinariedad.}

\begin{tabular}{|l|l|}
\hline $\begin{array}{l}\text { Interdisciplinariedad Estrecha } \\
\text { 1.Interacción entre disciplinas con el } \\
\text { mismo paradigma y método. }\end{array}$ & $\begin{array}{c}\text { 1. Interdisciplinariedad Ancha } \\
\text { paradigmas y métodos. }\end{array}$ \\
\hline 2.Pocas disciplinas involucradas. & 2. Muchas disciplinas involucradas \\
\hline $\begin{array}{c}\text { 3.Miembros de las disciplinas se } \\
\text { encuentran en la misma } \\
\text { organización. }\end{array}$ & $\begin{array}{c}\text { 3.Miembros de las diferentes organizaciones } \\
\text { son de diferentes áreas del conocimiento. }\end{array}$ \\
\hline $\begin{array}{c}\text { 4.Miembros de las disciplinas son } \\
\text { de la misma cultura. }\end{array}$ & $\begin{array}{c}\text { 4.Miembros de las disciplinas con de } \\
\text { diferentes culturas. }\end{array}$ \\
\hline
\end{tabular}

Fuente: (Dusseldorp D. v.).

\section{METODOLOGÍA}

En la Facultad de Ingeniería, las carreras históricamente se han caracterizado por desarrollar proyecto de vinculación de forma individual en diferentes ámbitos de la sociedad. Este es el caso de las carreras de Ingeniería Civil e Ingeniería Industrial, las cuales han sido las de mayor aporte a la Vinculación Universidad-Sociedad, Esto se menciona en diferentes artículos expuestos en el portal de internet de Presencia Universitaria (Vargas, 2014).

La Facultad de Ingeniería está conformada por seis disciplinas, que trabajan para el desarrollo tecnológico de la nación. En la Fig. 1 se muestra como estas carreras se pueden relacionar en un equipo de trabajo que logre grandes aportes a la sociedad desde la perspectiva o labor académica de la investigación, vinculación y docencia. Cabe mencionar que en el paradigma que se plantea a continuación, se involucra a las carreras de Ingeniería Agrónoma y Arquitectura, dado que se comparten los métodos y modelos utilizados en las ingenierías, generando una mayor afinidad y facilidad en la integración del equipo de trabajo. Es necesario visualizar que los brazos de apoyo de esta estructura deben ser la Dirección de Investigación Científica y Posgrado (DICYP) y la Dirección de Vinculación Universidad Sociedad (DVUS), para poder desarrollar todas las investigaciones, desarrollos tecnológicos y teorías que se pueden lograr recopilar en la intervención de múltiples equipos interdisciplinarios en las zonas rurales. 
Figura 1. Integración de los equipos interdisciplinarios de las carreras de Ingeniería y Direcciones de apoyo a la estructura de trabajo.

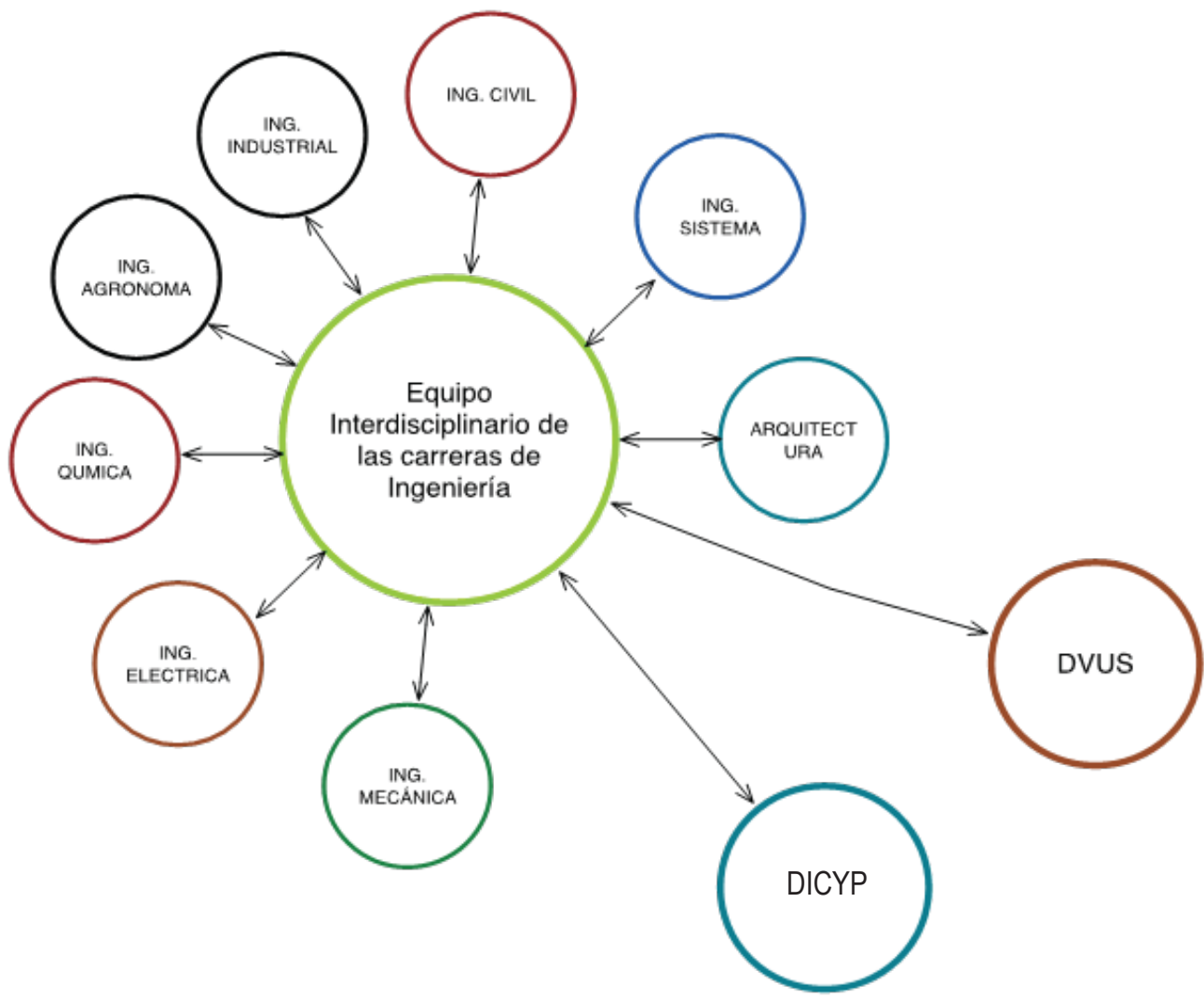

Fuente: Elaboración propia.

La metodología para cumplir con los objetivos de la investigación consta de 5 fases muy importantes: a) Integrar; b) Definir; c) Estructurar; d) Desarrollar; e) Evaluar la acción interventora del equipo interdisciplinario.

En la fase de integración se necesitó organizar al equipo de trabajo a través de las diversas carreras de la Facultad de Ingeniería, por medio de estudiantes que cumplieron con ciertas caracteristicas que los convierten en elegibles para pertenecer al equipo, cabe mencionar que los muchachos convocados fueron integrados para ser parte del equipo de manera voluntaria para que no dependan de una calificación en una asignatura determinada, sino de un proceso constructivista donde ellos crean su propio conocimiento sin olvidarse del enriquecimiento de su pensamiento crítico.

Para lograr seleccionar a los estudiantes, el método utilizado fue el Análisis de Proce- 
sos Jerárquicos (AHP) el cual ayuda a los tomadores de decisiones a encontrar la solución que mejor se ajusta a sus necesidades y a su comprensión del problema. Saaty (2009) planteó un método de poder seleccionar la mejor solución evaluando escenarios mediante la evaluación del nivel de importancia que tengan dichos factores o características del escenario.

Para la selección de los estudiantes, se tomaron en cuenta los aspectos relacionales y personales sugeridos por Llanos Paz (2012). Sin embargo, es necesario abundar en qué consisten esos aspectos personales y relacionales. En este caso se planteó un modelo de selección a través del método AHP ilustrado en la Fig 2. Aquí se logra visualizar como se relacionaron los criterios y sus subcategorías para la selección del candidato adecuado para el puesto.

Figura 2. Modelo AHP para la selección de candidatos para el puesto de miembro del aula campo.

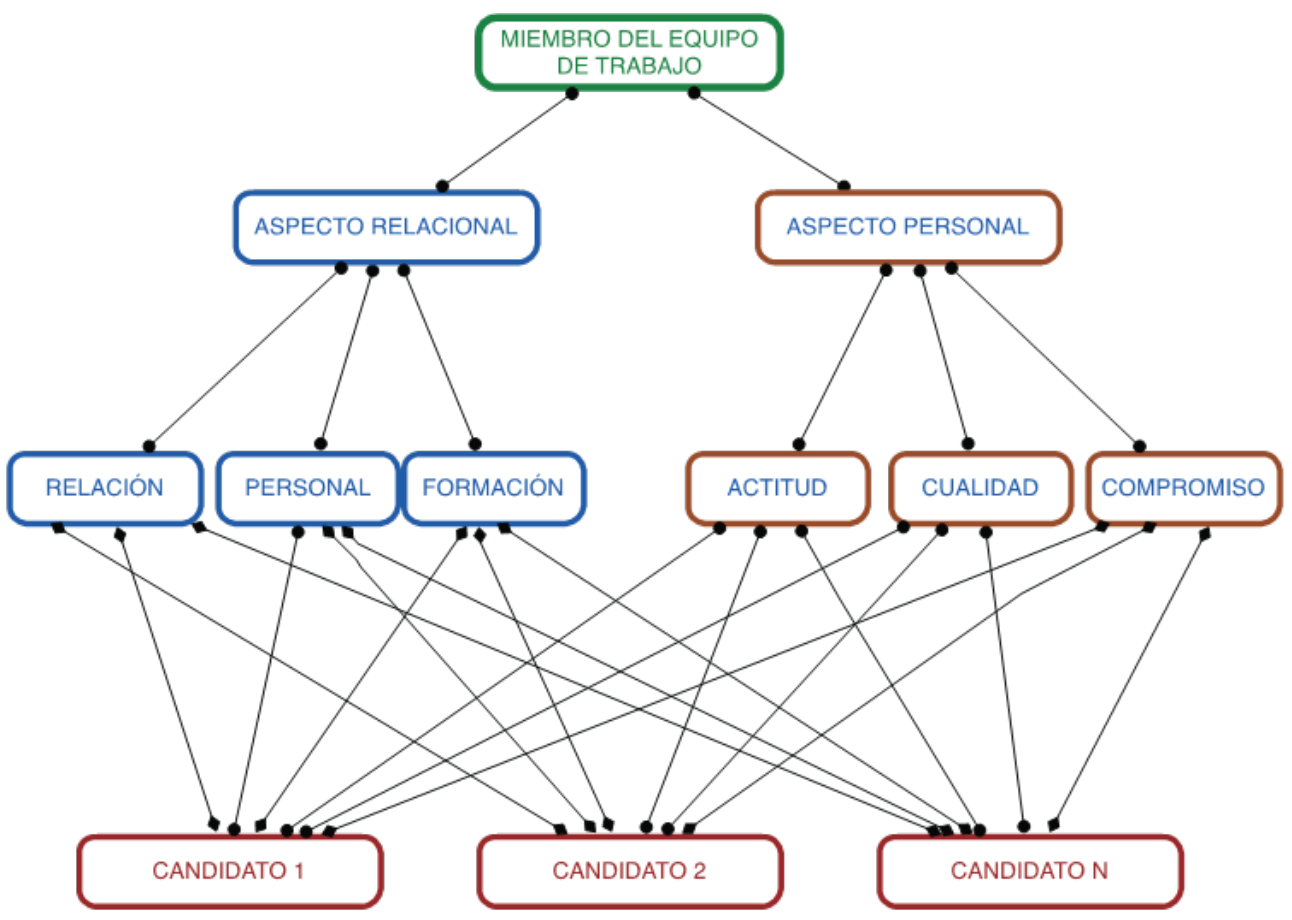

Fuente: Elaboración propia. 
Una vez seleccionado el equipo de trabajo, se desarrolló una agenda de trabajo de cinco meses donde debían resolver un problema mediante la metodología de aprendizaje basado en proyecto para intervenir la comunidad de Yamaranguila, departamento de Intibucá, Honduras. En esta parte, se define la labor del equipo para desarrollar las diferentes acciones interdisciplinarias a través de la opinión de los integrantes y de esta manera se utilizó nuevamente el método de AHP, para tomar la mejor decisión, los integrantes seleccionaron los criterios y definieron pesos para cada criterio.

Así mismo, el proyecto tiene varios hitos o puntos de evaluación importantes donde el estudiante tendrá la obligación de presentar varios entregables, como ser: a) Visitas de campo; b) Propuestas de ideas innovadoras y creativas; c) Diseño preliminar de ingeniería; d) Diseño final de ingeniería; e) Borrador de informes e f) Informe final. Para desarrollar todos estos entregables, es necesario que expertos en diversos temas brinden charlas, conversaciones o conferencias, para que los estudiantes logren aclarar muchos detalles sobre sus innovaciones.

Los proyectos seleccionados están integrados por los estudiantes de las diferentes carreras en una estructura matricial sólida para la ejecución, teniendo en cada proyecto un coordinador para trabajar en la gestión de todas las actividades del mismo. De esta manera, los miembros del equipo de trabajo tendrán coordinadores por área disciplinaria y por proyecto para solidificar sus labores dentro del equipo de trabajo. La organización del equipo interdisciplinario se muestra en la figura 3

\section{Figura 3. Matriz de Staff del Proyecto.}

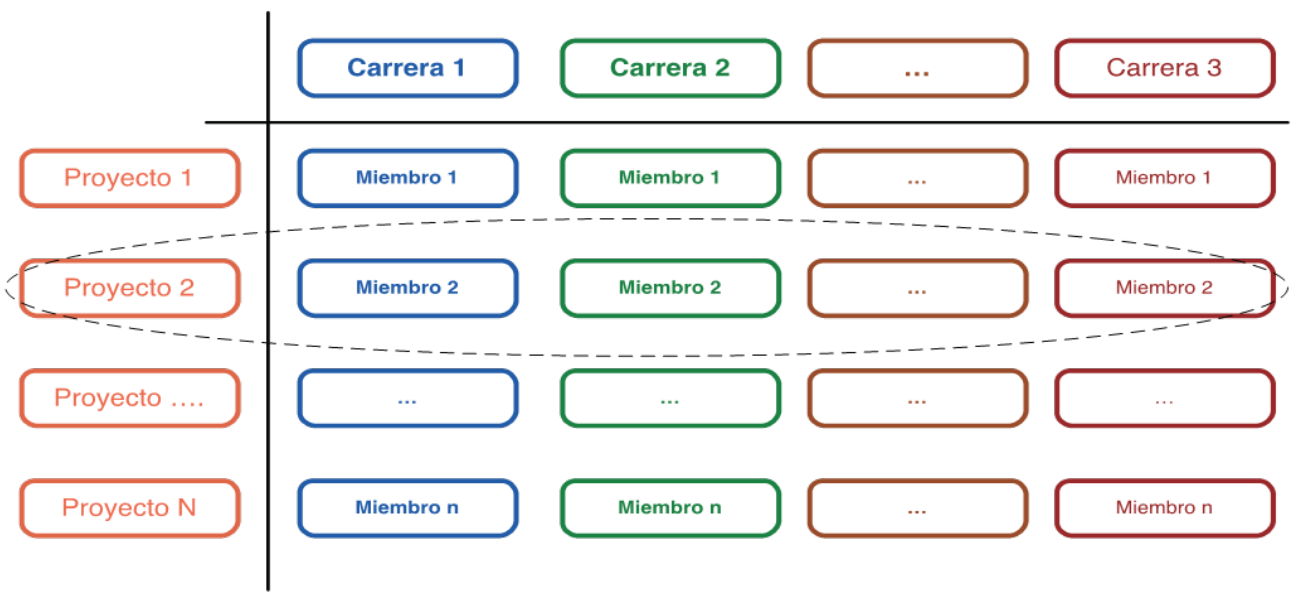

Fuente: Elaboración propia. 
El equipo de trabajo realizo varios diagnósticos para analizar la situación actual de los pueblos de la comunidad de Yamaranguila. Se seleccionó una comunidad cuya necesidad humana es muy grande. La aplicación de tres diagnósticos a lo largo de los seis meses que duro el proyecto se consideró importante para brindará información muy interesante respecto al aprendizaje de los estudiantes. Este diagnóstico ayudaron a visualizar la experiencia de la integración de las ingenierías los cuales fueron: a) Diagnostico de arranque; b) Diagnostico intermedio; y c) Diagnostico final.

\section{RESULTADOS}

En la etapa de integración, se seleccionó un grupo de doce personas para ser parte del equipo de trabajo que estaría interviniendo en el proyecto de Yamaranguila, el cual lo dirige la DUVS. En ese caso, seleccionaron dos personas para coordinar las áreas disciplinarias. Se utilizó el modelo AHP, siguiendo la ponderación mostrada en la Fig. 4.

Figura 4. Modelo AHP con la jerarquización de las características más importantes para la selección de los miembros del equipo de trabajo.

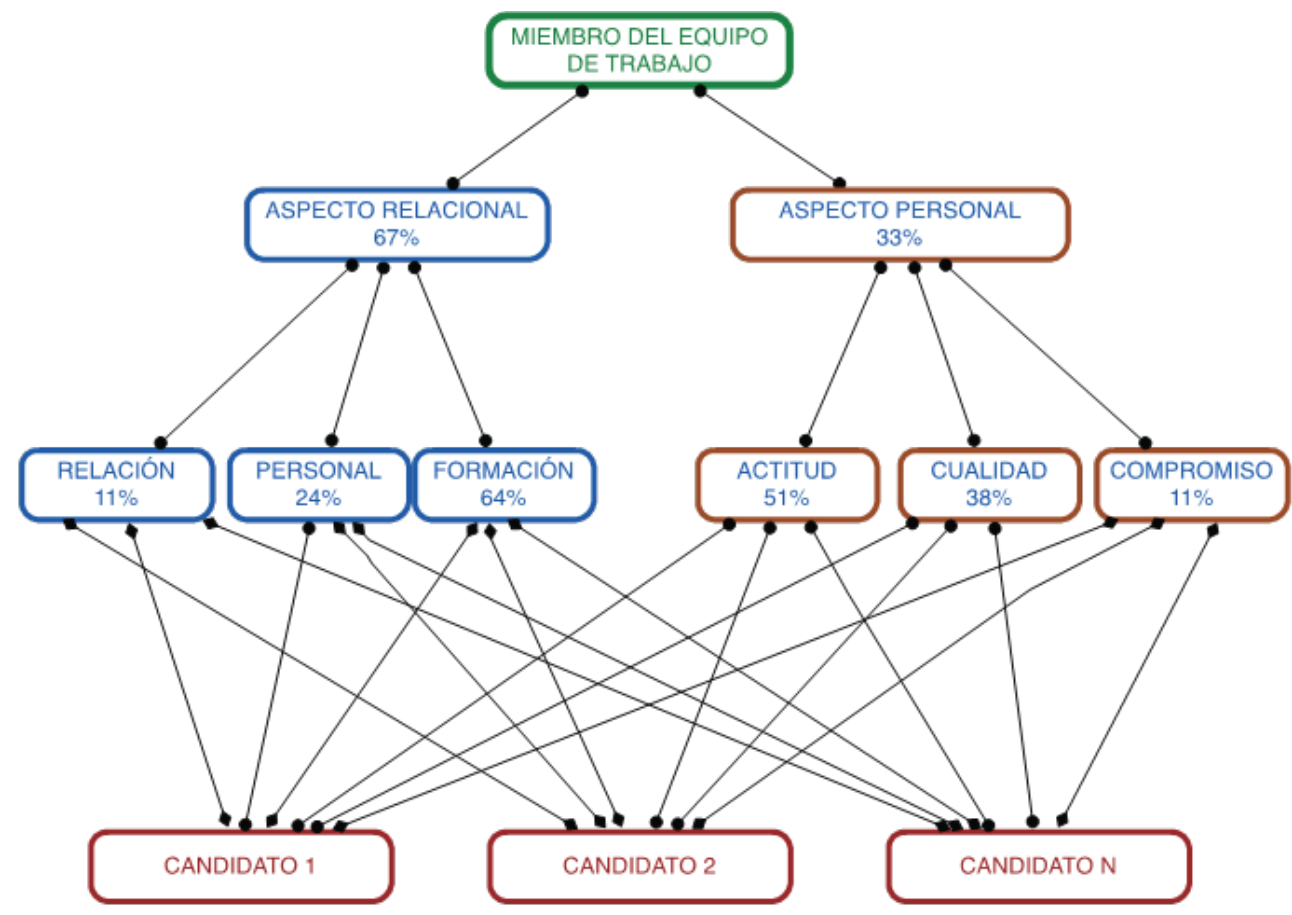

Fuente: Elaboracion Propia 
Los coordinadores se empoderaron en el equipo interdisciplinario de estudiantes de la Facultad de Ingeniería, quienes han logrado integrar a cuarenta personas activas, de las cuales se han tomado veinte y tres personas para que intervengan en el municipio de Yamaranguila. Al estar integrado los estudiantes, se comienza la etapa de definir la intervención del equipo de trabajo interdisciplinario en el proyecto, ligado a la carta de entendimiento de la UNAH con la Alcaldía del municipio de Yamaranguila. En esta se expresa que el apoyo universitario es fortalecer las capacidades de los pueblos indígenas del municipio. Con base a lo anterior, los coordinadores de área disciplinaria, con ayuda de su profesor tutor, desarrollaron un plan de reconocimiento para visualizar las necesidades y carencias que tienen en el municipio de Yamaranguila en términos de ingeniería.

En la primera visita al municipio, el equipo interdisciplinario visitaron las siguientes 4 aldeas aledañas a la comunidad de yamaranguila centro: a) El Pelón; b) Planes de Pacaya; c) Carrizal Semane; y d) el barrio Buenos Aires. En dicho lugares, se realizaron encuestas para determinar cuál de ellas se encuentra con mayor necesidad a nivel de ingeniería. En el primer informe o diagnostico brindado por el equipo de trabajo, se manifiesta la necesidad de intervención en la comunidad de Carrizal Semane, una comunidad vulnerable ante eventos naturales, en especial terremotos y deslizamientos de tierra.

En Carrizal Semane cuentan con una cantidad promedio de 5 habitantes por casas según las encuestas aplicadas. Los rubros económicos principales de la comunidad son la agricultura y el comercio, la gráfica 1 muestra que los cultivos predominantes son el plátano, café y maíz. Los pobladores de la zona manifestaron su preocupación con respecto a la seguridad alimentaria, ya que el suelo no permite la producción deseada y los factores climatológicos agravan aún más la situación. Cabe mencionar que Carrizal Semane se localiza en la zona baja de Yamaranguila, siendo más afectado por las sequías.

En la actualidad, la comunidad cuenta con servicio de agua potable en un $84 \%$ de las viviendas, de acuerdo a las encuestas aplicadas; se asume que la fuente no brinda la calidad de agua necesaria para el consumo. Los pobladores pagan L30.00 mensuales por el servicio de agua para el consumo humano, animal y el riego de cultivos.

A nivel de vivienda, se indicó que existen 35 casas en la comunidad y la mayoría de ellas presenta fisuras por la inestabilidad en el suelo de la zona y por algunas deficiencias constructivas que se identificaron. En la gráfica 2 se muestra que el $64 \%$ de las casas se encuentran en estado regular, para determinar esto se tomó en consi- 
deración el aspecto estructural de la vivienda, entre los parámetros se encuentran; fisuras en las paredes, estado del techo, posee chimenea, tipo de piso, aspectos arquitectónicos, por lo que en algunos casos las personas ya dejaron de vivir en su casa y utilizan otras estructuras para habitar. La gran mayoría de casas se encuentran construidas de paredes de adobe y techo de teja Así mismo, la comunidad no cuenta con energía eléctrica, Solamente el $12 \%$ de la comunidad tiene acceso a energía mediante la utilización de paneles solares o generadores de combustión interna. No se cuenta con una red de saneamiento y el uso de letrinas es limitado.

\section{Gráfica 1. Tipos de cultivos}

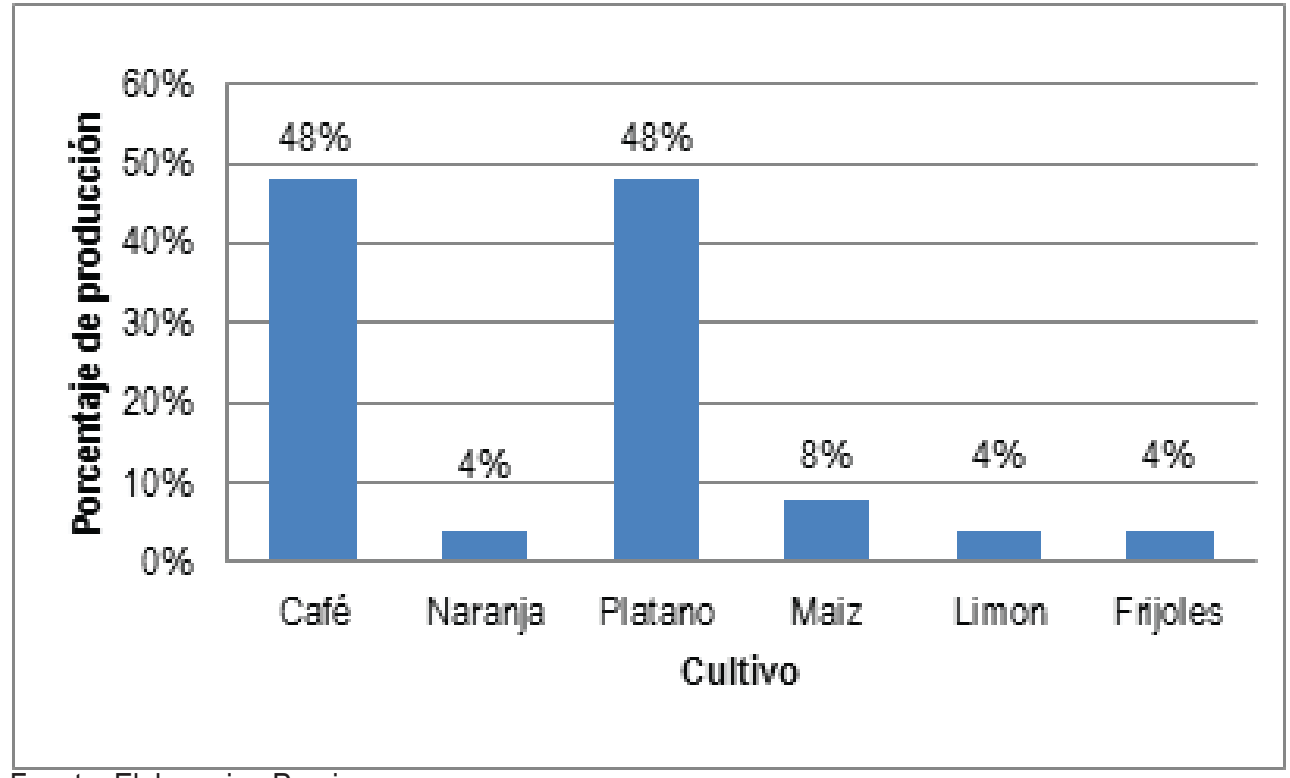

Fuente: Elaboracion Propia

Adicionalmente, se analizó la situación de los desastres naturales en la zona, y se determinó que los movimientos de laderas y desprendimientos de rocas son las mayores amenazas. Se mencionan en menor medida las lluvias fuertes, temblores y vientos huracanados. Los pobladores coinciden en afirmar que sus viviendas no han sido reparadas después un desastre natural, y esto obliga a las familias a vivir en condiciones muy peligrosas.

Con base en los hallazgos de la primera visita, el equipo de trabajo planteó una estructura para el desarrollo del proyecto, la cual se enfoca en las necesidades que tiene la zona actualmente y que se encuentre enmarcada en el proceso del fortalecimiento de las capacidades de los pueblos indígenas. Estas necesidades son: a) Vivienda; b) Centro de salud; c) Accesos; d) Vulnerabilidad; e) Agua potable y sanea- 
miento; y f) Sistema de información en la alcaldía. En la Tabla 2 se logra observar cómo se abordan los temas prioritarios según la integración de las carreras.

Gráfica 2. Condición de viviendas

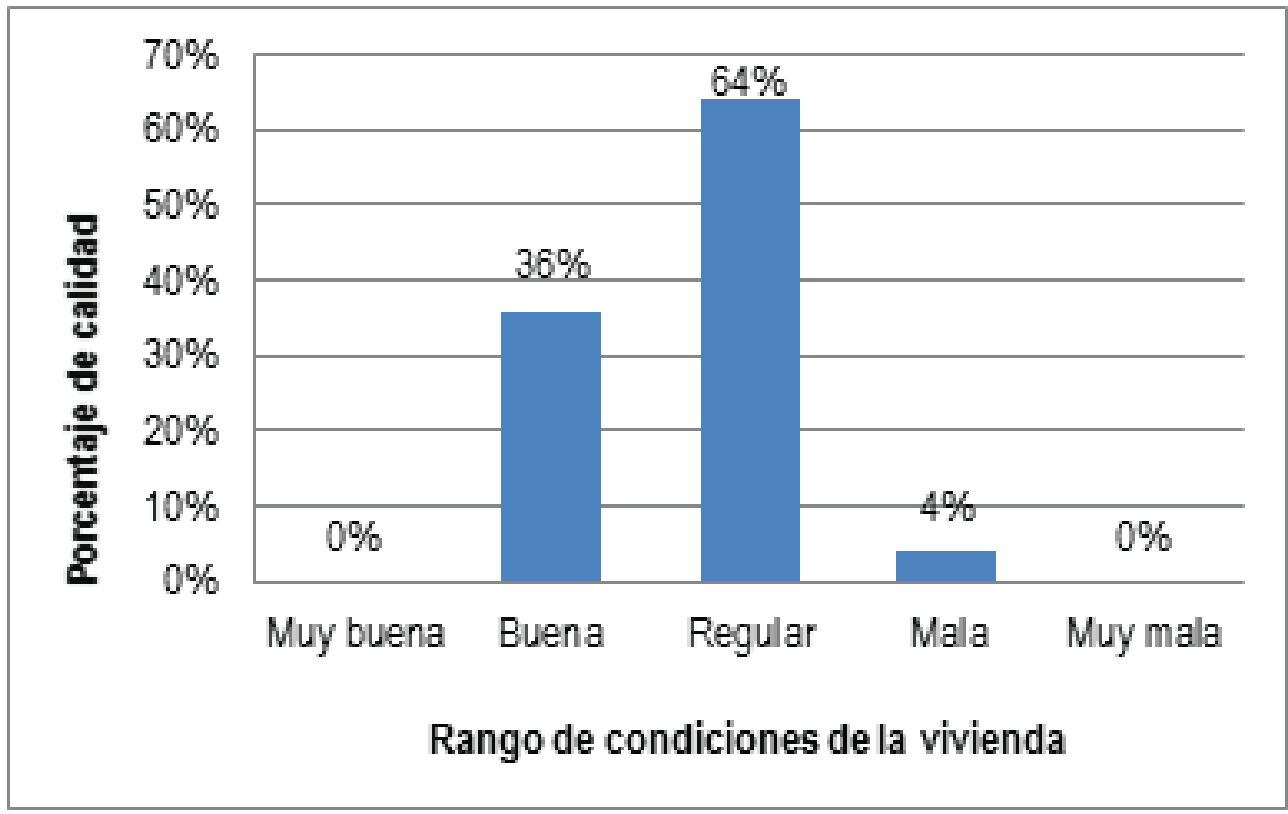

Fuente: Elaboracion Propia

Tabla 2. Matriz de staff para desarrollo de proyectos prioritarios en la zona.

\begin{tabular}{|l|c|c|c|c|c|c|c|}
\hline \multirow{2}{*}{ Prioridad } & \multicolumn{7}{c|}{ Carrera } \\
\cline { 2 - 9 } & Civil & Mecánica & Eléctrica & Sistemas & Industrial & Química & Arquitectura \\
\hline Casa & $\mathrm{x}$ & $\mathrm{x}$ & $\mathrm{x}$ & & $\mathrm{x}$ & $\mathrm{x}$ & $\mathrm{x}$ \\
\hline Centro de salud & & $\mathrm{x}$ & $\mathrm{x}$ & & & & $\mathrm{x}$ \\
\hline Accesos & $\mathrm{x}$ & & $\mathrm{x}$ & & & & \\
\hline Vulnerabilidad & $\mathrm{x}$ & & & & $\mathrm{x}$ & & \\
\hline $\begin{array}{l}\text { Agua potable y } \\
\text { saneamiento }\end{array}$ & $\mathrm{x}$ & $\mathrm{x}$ & & & & & \\
\hline $\begin{array}{l}\text { Desarrollo de la } \\
\text { informática de la } \\
\text { Alcaldía }\end{array}$ & & & & & & & \\
\hline
\end{tabular}

Fuente: Elaboracion Propia 
En la cuarta etapa de desarrolló, se visualizó que los equipos interdisciplinarios juegan un rol por proyecto integrado por las diferentes carreras. Se planificó en los meses de marzo a septiembre del año 2015 tiempo que corresponde al segundo periodo académico, las actividades a desarrollar en la segunda gira a la comunidad de Carrizal Semane. La Tabla 3 muestra los objetivos de cada proyecto.

\section{Tabla 3. Objetivos de la segunda visita a Carrizal Semane.}

\begin{tabular}{|c|c|}
\hline Proyecto & \multicolumn{1}{c|}{ Objetivos } \\
\hline \multirow{4}{*}{ Casa } & $\begin{array}{l}\text { 1. Recolectar información más detallada de las casas, como } \\
\text { ser: distribución arquitectónica, tipo de piso, estado del } \\
\text { techo, condición estructural. }\end{array}$ \\
\hline 2. Medir terreno donde se instalará el prototipo de vivienda \\
3. Analizar materiales de la zona.
\end{tabular}

Fuente: Elaboracion Propia

La segunda visita fue realizada con la participación de veinte estudiantes de las diferentes carreras de ingeniería y se se integraron dos estudiantes de arquitectura y dos de microbiología. Las actividades fueron desarrolladas siguiendo el cronograma del proyecto a ejecutar. En la imagen 1 se observa el trabajo de los muchachos en la zona de Carrizal Semane. En esta visita se hizo una inspección más a fondo de la comunidad seleccionada, cada equipo levanto la información que necesitaba. 
Imagen 1. Equipo interdisciplinario en zona: a) Equipo de trabajo saliendo de la escuela; b) Analizando residuos de los hogares; c) de Recorrido a la toma de agua.
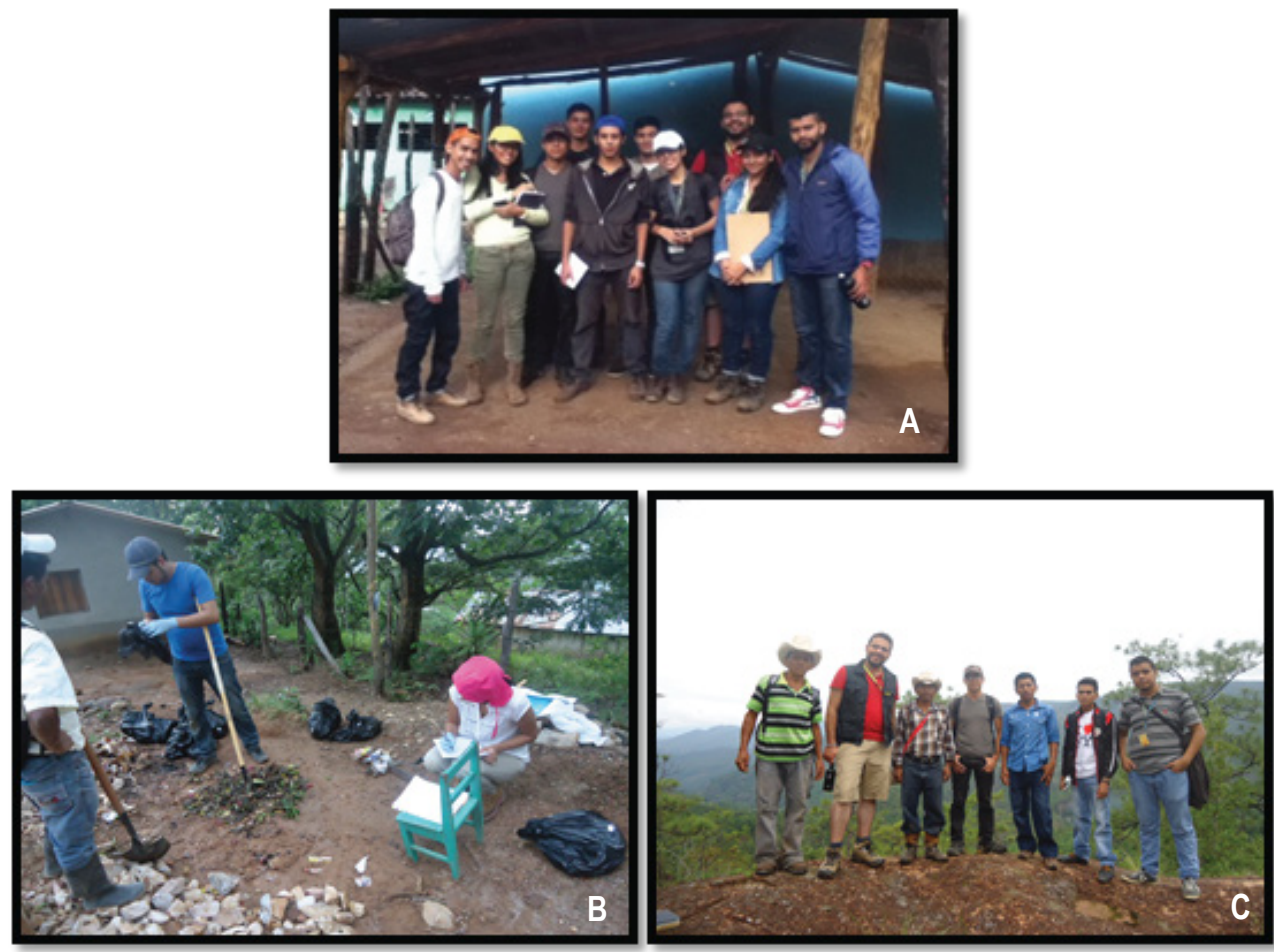

Fuente: Elaboracion Propia

\section{DISCUSIÓN}

Conformar equipos interdisciplinarios es un reto, en especial cuando se ha venido trabajando de manera disciplinaria durante mucho tiempo. Se debe lograr que los estudiantes analicen las situaciones, no solo desde la perspectiva de su campo de estudio, sino que tengan una visión más holística de lo que se le plantea que resuelva. El hecho que al estudiante se le asignen problemáticas reales para ser resueltas le ayuda a entender mejor cómo será su función como egresado. En el caso de proyectos de carácter social, se debe tener clara la metodología de trabajo, dado que el éxito del proyecto va a depender mucho de la aceptación e involucramiento de las personas beneficiadas. El equipo de profesionales que intervengan la zona debe saber cómo dirigirse a los pobladores, saber cómo incluirlos en la planificación, saber escuchar y aprender. 
Sin duda, esto hace profesionales más humanos y conscientes de la realidad del país en el que viven, ya que conocen de primera mano la precariedad y dificultades a las que se enfrentan día a día las personas que habitan en las zonas rurales y comunidades indígenas. Otro aspecto importante es el hecho de comprender que los proyectos se deben adaptar a ciertos parámetros inherentes a la zona donde se influirá, como por ejemplo, religión, arquitectura, costumbres y creencias. Esto evitará alterar la esencia de la comunidad.

La falta de presupuesto es una de las principales limitantes del proyecto puesto que es necesario movilizarse a zonas usualmente retiradas, llevar equipo, material, entre otras cosas que generan gastos.

No todos los maestros están de acuerdo con nuevas estrategias de enseñanza, por lo que se dificulta la obtención de asesores temáticos para validar el correcto aprendizaje dentro de los proyectos planteados en los equipos interdisciplinarios. Los profesores deben estar plenamente comprometidos para que se puedan brindar el asesoramiento correcto dentro del espacio académico correspondiente.

Esta metodología puede implicar una mejor abstracción dentro de las aulas de clases para involucrar a diferentes áreas de la ciencia en la búsqueda de soluciones más atractivas para la sociedad. Dentro de las carreras de ingeniería se encuentran las clases de proyecto de ingeniería, las cuales se pueden unir para formar equipos interdisciplinarios para afrontar problemas dentro de la clase. Así mismo, la metodología puede ser replicada para las diferentes áreas del quehacer universitario como lo es la vinculación y la investigación científica.

\section{CONCLUSIONES}

El trabajo realizado a lo largo de la comunidad de Carrizal Semane, municipio de Yamaranguila, Departamento de Intibucá por parte del equipo interdisciplinario de la Facultad de Ingeniería hace constar que la integración de las diferentes carreras es posible para brindar las soluciones necesarias para el fortalecimiento de las capacidades de estos pueblos indígenas. La ingeniería, como área del conocimiento en la UNAH, puede definir acciones interdisciplinarias reales para clarificar la gestión de apoyo con la comunidad, estructurando su intervención con base en las áreas disciplinarias para desarrollar actividades y brindar soluciones a la problemática que 
tienen estos pueblos indígenas. Es necesario seguir adelante con el proyecto para lograr culminar con la etapa de desarrollo y evaluación.

\section{BIBLIOGRAFÍA}

Doldan Tie, F. (2003). Dirección Financiera de la Empresa. Santiago: Torculo.

Gerbhard, A., \& Zemelman, M. (1979). Orientación Vocacional para la Juventud. Santiago, Chile: Andres Bello.

Gil-Perez, D., \& Vilches, A. (2014). Ciencia de la sostenibilidad. (U. d. Cádiz, Ed.)

Revista Eureka sobre Enseñanza y Divulgación de las Ciencias, 11(3), 1-3.

Llanos Paz, F. D. (2012). La sostenibilidad de Equipo de voluntario: Una evaluación

objetiva mediante la aplicación del modelo AHP en asociaciones juvenieles no lucrativas de ocio y rimpo libre. Caso Gallego. Revista de Economía Pública, Social y Cooperativa(74), 59-85.

Marques, J. (2008). Inter and multidisciplinarity in engineering education. Int. J Mech DEs, 4, 229:237.

Morillo, F., Bordons, M., \& Gómez, I. (2003). Interdisciplinarity in Science: Atentative type disciplines and research areas. . Journal of the American Society for information Science and Technology., 54(13), 1237-1249.

Morin, E. (1999). Los sietes saberes necesarios para la educación del futuro. (O. d. Unidas, Ed.) Educación, la Ciencia y la Cultura .

Ortega y Gasset, J. (1960). Misión de la Univerisdad y otro ensayos (3 ed.). Revista de Occidente.

Saaty, T. L., \& Katz, J. M. (1990). How to make a decision: The Analityc Hierarchy Process. European Journal of operacional Research, 38, 9-26.

UNAH. (2014). Lo esencial de la Reforma Universitaria: Ética, fortalecimiento de la identidad nacional y gestión cultural para la construcción de la ciudadanía. Tegucigalpa, Honduras: Editorail Universitaria UNAH.

UNAH. (2009). Modelo Educativo de la UNAH. Tegucigalap, HOnduras: Editorial Univeristaria UNAH.

UNAH. (2008). Reglamento de Departamento y Carreras de la UNAH. (L. Gaceta, Ed.) Recuperado el 2015, de Leyes y Reglamentos de UNAH: https://sg.unah.edu.hn/nuevo-pagina-2/nuevo-pagina/\#document-panel28

Van de Besselaar, P., \& Heimerik, G. (s.f.). Dsiciplinarity, multidisciplinary, interdisciplinary: Concepts and indicators. Proceeding 8th conference on Scientimetrics and Informetrics. 2001, págs. 705-716. Sydney: M. Davis \& C.S Wilson.

Van Dusseldorp, D., \& Wigboldus, S. (s.f.). Interdisciplinary Research for integrated 
rural develpoment in developing countries: The Role of Social Sciences. Issues in Integrative Studies(12), 93-138.

Vargas, Y. (2014). Estudiantes de ingeniería industrial fabricarán carros cuneros para el materno infantil . Presencia Universitaria UNAH.

Vargas, Y. (2014). Ingenieros de la UNAH planifican proyectos de desarrollo en Oropoli. Presencia Universitaria UNAH.

Vargas, Y. (2014). Ingeniero de la UNAH planifican proyectos de desarrollo en Oropoli. Presencia Universitaria UNAH.

Vargas, Y. (2014). Presencia Universitaria UNAH. 